WIENER SLAVISTISCHES JAHRBUCH, Band 55/2009, 97-107

(C) 2009 by Österreichische Akademie der Wissenschaften, Wien

BORISLAV PETRANOV

\title{
Das bulgarische Drama außerhalb Bulgariens im 21. Jahrhundert
}

In der Entwicklung des bulgarischen Dramas zu Beginn des 20. Jahrhunderts können dieselben Charakteristika beobachtet werden, die auch für die Entwicklung des europäischen Dramas dieser Periode typisch sind. Von besonderem Interesse sind die Theaterstücke, die in den 20er Jahren entstanden sind. Thematik und Struktur dieser Werke decken sich mit den Fragestellungen der modernen europäischen Autoren aus jener Zeit. Es gibt Autoren, die vom Symbolismus beeinflusst worden sind, aber auch solche, in deren Stücken man den Nachhall des Naturalismus vernehmen kann. Im Allgemeinen entwickelt sich das bulgarische Drama bis 1944 in verschiedenste Richtungen, und gerade in dieser Vielfalt liegt eine seiner größten Stärken.

In den Jahren nach dem September 1944 wird den Dramatikern das für die Literatur in der Sowjetunion charakteristische Modell des sozialistischen Realismus aufgezwungen. In Bulgarien wird nicht nur das autoritäre politische System des sozialistischen Imperiums durchgesetzt, sondern es wird auch jegliches Andersdenken in der Literatur verboten. Während der 70er und 80er Jahre des vergangenen Jahrhunderts erschienen die ersten Theaterstücke, die mithilfe der allegorischen Sprache der Fabeln versuchten, die akut gewordenen Probleme in der Entwicklung des sozialistischen Bulgariens zu thematisieren. Die Inszenierungen solcher Texte erfreuten sich eines außergewöhnlichen Interesses seitens der Zuschauer. Diese Theaterstücke wurden von staatlicher Seite verboten und von den Bühnen verbannt. All das trug zu ihrer Umhüllung mit einer besonderen Aura bei - ihre Autoren wurden zu Helden. In seiner Entwicklung verpasste das bulgarische Theater während dieser Periode eine ganze Etappe, insbesondere das Aufkommen und die Entwicklung des absurden Dramas. Zwar tauchten auf einigen alternativen Bühnen Interpretationen von Texten Eugène Ionescos auf, doch nur sporadisch, so dass eigentlich nicht nur in der Dramatik, sondern auch in der dramaturgischen Praxis Erfahrungen in der Deutung dieser Art von Stücken fehlten. 
Im untersuchten Zeitabschnitt aus der zweiten Hälfte des 20. Jahrhunderts fand die bulgarische Dramatik auch außerhalb der Grenzen des Landes durchaus Verbreitung. Das Erscheinen von Anthologien und selbständigen Ausgaben des Schaffens bestimmter Dramatiker sind keine Seltenheit. Aber hier muss ich gleich hinzufügen, dass das meistens im Rahmen des so genannten „Sozialistischen Lagers“ passierte. Auf diese Weise erschienen in der ehemaligen DDR zahlreiche Veröffentlichungen von bulgarischen Theaterstücken in deutscher Sprache. Ebenso ist das bulgarische Drama auch in russischer, polnischer, tschechischer, slowakischer und ungarischer Sprache vorgestellt worden. Man muss hervorheben, dass diese „Invasion“ vor allem das Ergebnis einer gezielten ideologisch-nationalen Kulturpolitik war. In jener Zeit stellte der Staat Mittel zur Popularisierung der bulgarischen Dramatik außerhalb des Landes zur Verfügung, selbstverständlich vorwiegend in den sozialistischen „Bruderstaaten".

Nach der politischen Wende von 1989 fielen alle Tabus. Das Publikum erwartete, dass in den Schubladen der „Helden“ viele Texte lägen, die nur auf ihre Premiere warteten. Bedauerlicherweise erwies sich das Theater auf der Straße als viel interessanter als jenes auf den Bühnen. Die allegorische Sprache der Fabeln hatte ausgedient. Auf den Bühnen hielt Abgeschmacktheit Einzug. Im Kampf um das Publikum entbrannte eine Schlacht der Ausdrucksmittel. Manche Regisseure überschritten in ihrer Machtlosigkeit die Grenze des Ästhetischen und gelangten zu offenkundiger Pornographie. Andere versuchten, der Straße Konkurrenz zu machen, indem sie politische Spektakel veranstalteten. Aber das reale Theater draußen war stärker. Der Anfang der 90er Jahre war eine Periode der Collagen in der Dramatik. Niemand mehr griff nach einem ganzen Theaterstück. Jeder zog es vor, irgendeinen Text neu zu variieren und zu vervollständigen. Genau in diese Zeit fallen auch die ersten Gehversuche neuer Namen der bulgarischen Dramatik. Doch sie kamen nicht aus dem Nichts. Ihre Arbeit ist ein Produkt des Anhäufens und des Zusammenführens von Kenntnissen und Erfahrungen. Auf eine natürliche Art und Weise kehrte so die Vielfalt in die bulgarische Dramatik zurück.

Gegenstand dieser Untersuchung sind die am Beginn des 21. Jahrhunderts herausgegebenen Anthologien bulgarischer Theaterstücke. Bedauerlicherweise erschien zwischen den Jahren 1989 und 2000 kein einziger Band mit bulgarischer Dramatik in einer fremder Sprache. Die Periode des politischen, ökonomischen und anderer Arten des Übergangs wirkte sich auch auf die Popularisierung der bulgarischen Dramatik aus. Man bemerkte Interesse, aber es wurden nur Texte für konkrete Anlässe übersetzt - wenn das entsprechende Stück in den Spielplan irgendeines Theaters aufgenommen wurde.

Die Anthologien, welche ich vorstellen möchte, erschienen auf dem internationalen Markt von 2002 bis 2006. Ich zähle sie in der Reihenfolge ihres Erscheinens auf: 
2002:

„Zeitgenössisches bulgarisches Drama“, Band 1 („CONTEMPORARY BULGARIAN DRAMA 1") - in englischer Sprache, Ausgabe der Vereinigung der bulgarischen Theaterkritiker „Antrakt“;

„Zeitgenössische bulgarische Bühnenstücke“ („CONTEMPORARY BULGARIAN PLAYS“) - in englischer Sprache, Ausgabe des Verlags „Tantalus Books“, Großbritannien;

2003:

„Zeitgenössisches bulgarisches Drama“, („САВРЕМЕНА БУГАРСКА ДРАMA“) - in serbischer Sprache, Ausgabe des Nationaltheaters in Belgrad aus der Reihe „Die Balkan-Bühnenstücke Europas“, Serbien;

„Der Oberst Vogel und andere Stücke“ (,THE COLONEL BIRD AND OTHER PLAYS“) - in englischer Sprache, Ausgabe des Verlags „Tantalus Books“, Großbritannien;

2004:

„Zeitgenössische bulgarische Dramatik“, Band 1 („СОВРЕМЕННАЯ БОЛГАРСКАЯ ДРАМАТУРГИЯ - ВЫПУСК І“) - in russischer Sprache, Ausgabe des Bulgarischen Kultur- und Informationszentrum und der Zeitschrift „Петербургский театралный журнал“, Russland;

2006:

„Bulgarische Dramen“, („BULGAR OYUNLARI“) - in türkischer Sprache, Ausgabe des Verlags „Papirüs“, Türkei;

„Junges Theater aus Bulgarien“, (,JUNGES THEATER AUS BULGARIEN“) in deutscher Sprache, Ausgabe des Verlags „Drava“, Österreich.

Um das Interesse an der bulgarischen Dramatik der letzten zehn Jahre des 20. Jahrhunderts zu wecken und auch um die jungen bulgarischen Dramatiker der Welt zu präsentieren, gab die Vereinigung der bulgarischen Theaterkritiker „Antrakt" den ersten Band der Anthologie „Zeitgenössisches bulgarisches Drama“ („CONTEMPORARY BULGARIAN DRAMA“) in englischer Sprache heraus. Die Herausgeber erläutern ihre Absichten in den kurzen Begleittexten zu den herausgegebenen Bühnenstücken. „Das ist der erste Band einer Reihe, welche die zeitgenössischen bulgarischen Stücke vorstellt.“ (Panova - Jordanov - Nikolova 2002: 4). Das Redaktionskollegium wurde aus bedeutenden Theoretikern des bulgarischen Theaters zusammengestellt - Prof. Dr. Snežina Panova, Doz. Dr. Nikolaj Jordanov, Doz. Dr. Kamelia Nikolova, Dr. Agelika Stefanova und die junge Regisseurin Elena Panajotova. Die Herausgeber erklären deutlich die Kriterien, nach denen die Autoren und ihre Stücke ausgewählt wurden: „... die sechs bulgarischen Theaterstücke in diesem Band wurden auf den Bühnen des bulgarischen Theaters in der Saison 2001/2002 inszeniert oder sind im Prozess der Inszenierung." (Panova - Jordanov - Nikolova 2002: 4). Sie unterstreichen als Grundprinzip der Auswahl auch die Qualität der angebotenen Texte. Die Anthologie umfasst sechs bulgarische Dramatiker, deren Na- 
men in den 90er Jahren des 20. Jahrhunderts auftauchten und sich durchsetzten. Der Schauspieler und Dramaturg Kamen Donev ist mit dem Stück „Das Flugzeug Flüchtling" aufgenommen, Elin Rahnev - eine der großen Hoffnungen der bulgarischen Theaterlandschaft zu jener Zeit - mit dem Stück „Flaubert“. Anna Topaldžikova ist eine bekannte Theoretikerin und Kritikerin des bulgarischen Theaters; sie sowie Jurij Dačev - auch ein bekannter Theaterkritiker mit scharfsinniger Schreibweise - „betraten das Theater durch den Diensteingang“. Topaldžikova wird mit ihrem Stück „Widerspiegelungen“ vorgestellt, Dačev mit dem Drama „Salon der Tränen“. Die Palette wird mit zwei weiteren Frauen bereichert - Teodora Dimova, ein bereits anerkannter Name in der jungen bulgarischen Dramatik mit „Neda und die Hunde" und Ina Božidarova, die gerade ihre ersten Schritte im Stückeschreiben macht, mit „Aschenputtel - Bühnenvariationen“. Die Ausgabe dieses Sammelbandes war die erste Geste der Fürsorge für die neu aufgetauchten Autoren im bulgarischen Theater. Haupteigenschaft der Anthologie ist die Präsentation der Genrevielfalt der Texte. Ein interessantes Forschungsobjekt sind die Übersetzungen. Nur zwei der Bühnenstücke wurden von jemandem übersetzt, dessen Muttersprache Englisch ist, nämlich von David Mossop. Alle anderen Werke wurden von sehr talentierten Übersetzern aus dem Bulgarischen ins Englische übertragen - Iskra Nikolova, Dimana Ilieva, Elena Aleksieva und Kornelia Slavova. Deshalb ist eine der wichtigsten Fragen, die bei der Analyse der Qualitäten der Anthologie aufkommt, jene, inwieweit diese Übersetzungen in der englischsprachigen Welt verwendbar sind. Angesichts dessen, dass der Band zum Zweck der Präsentation des bulgarischen Dramas außerhalb Bulgariens herausgegeben wurde, denke ich, dass dieses Ziel erreicht wurde. Was die Frage des Vertriebs betrifft, so fand ein solcher im wahrsten Sinne dieses Wortes mit dieser Ausgabe nicht statt. Das Bändchen wurde von Leuten, die sich mit Theater beschäftigen, verschenkt und verteilt. Das heißt, die Anthologie hatte eher einen Werbewert. Die Idee war gut und wurde auch gut verwirklicht, aber leider blieb dieser Band 1 auch der einzige Band. Ich vermute, dass der Grund, weshalb die Herausgeber ihre Idee nicht weiterentwickelten, die spätere Veröffentlichung von Sammelbänden in anderen Sprachen war.

Im selben Jahr 2002 brachte der englische Verlag „Tantalus Books“ die Anthologie „Zeitgenössische bulgarische Bühnenstücke“ auf den Buchmarkt. Herausgeber des Sammelbandes sind Anna Karabinska und Josepha Jacobson. Das ist die erste Ausgabe bulgarischer Bühnenstücke von einem britischen Verlag sowie die erste Anthologie bulgarischer Dramatik, die auf den Buchmärkten im englischsprachigen Raum vertrieben wird. Besondere Aufmerksamkeit muss den Übersetzungen gewidmet werden, welche von den besten Übersetzern aus dem Bulgarischen ins Englische angefertigt wurden - Anna Karabinska, Judith Sprostranova, Thomas Crowe, Josepha Jacobson. Der Band enthält die Stücke „Nirvana“ von Konstantin Iliev, „Januar“ von Jordan Radičkov, „Linienbus“" von Stanislav Stratiev und „Die Menschenfresserin“ von Ivan Radoev. Ich gestatte mir aber den Titel der Anthologie „Zeitgenössische bulgarische Bühnenstücke“ in Frage zu stellen, weil alle vier Texte Ende 
der 70er und Anfang der 80er Jahre des 20. Jahrhunderts geschrieben wurden. Sie sind schon seit langer Zeit in der Rubrik ,zeitgenössische Klassik“ der bulgarischen Dramatik zu finden. Alle vier Stücke wurden unter großem Publikumsinteresse nicht nur auf bulgarischen Bühnen, sondern auch auf den Bühnen der europäischen „Bruderstaaten“"gespielt. Radičkov, Stratiev und Radoev sind bereits verstorben und so zu echten Klassikern der bulgarischen Literatur geworden. Erfreulicherweise verfasst Konstantin Iliev neue Theatertexte, die weiterhin Regisseure und Publikum begeistern. Ein Beispiel dafür ist sein Stück „Francesca“, welches in einer der Anthologien, die wir hier besprechen, zu finden ist. Als interessanten Zug der Persönlichkeit Konstantin Ilievs muss man erwähnen, dass er 1996 in Wien den Herderpreis für sein Lebenswerk erhielt. Das Stück „Nirvana“ aus diesem Sammelband fand eine interessante Aufnahme in England. Es wurde auf einem der prestigeträchtigsten Bewerbe für Übersetzer von Bühnenstücken in London - „Revelations: The Gate Translations Award“ - aus 73 Übersetzungen mit weiteren fünf Texten für das Finale nominiert. Dank der Veröffentlichung des Stücks in der von „Tantalus Books“ herausgegebenen Anthologie wurde es in London uraufgeführt. „Vom 1. bis zum 20. März um 20.00 Uhr kann man in den ,Riverside Studios' in London das Stück ,Nirvana' von Konstantin Iliev sehen ... Ich kenne keine ermutigendere Beurteilung des bulgarischen Theaters als diese, in einem so angesehenem Theaterzentrum wie London bulgarische Bühnenstücke zu inszenieren. ,Riverside Studios ' ist eines der besten Kulturzentren in London mit speziellen Interessen an internationalen Projekten ..., Nirvana' ist das erste bulgarische Bühnenstück, das in West End inszeniert wurde.“ (Nirvana 2005) Der Sinn der Herausgabe von Anthologien mit bulgarischen Stücken ist es, die Bühnen in den jeweiligen Ländern zu erreichen. In dieser Hinsicht sind die ,Zeitgenössischen bulgarischen Bühnenstücke“ des Verlags „Tantalus Books“ ein herrliches Beispiel für den Zusammenhang zwischen Publikation und Intention eines Textes.

2003 wurde eine Anthologie mit zeitgenössischen bulgarischen Bühnenstücken herausgegeben, die ebenfalls einzigartig ist. Der Sammelband „Zeitgenössisches bulgarisches Drama“, („САВРЕМЕНА БУГАРСКА ДРАМА“) wurde in serbischer Sprache veröffentlicht. Er erschien in der Reihe „Die Balkan-Bühnenstücke Europas“" und ist eine Ausgabe des Nationaltheaters in Belgrad. Verfasser sind Želko Hubač und Emil Bonev. Übersetzt wurden die Texte von Blagoe Nikolić. In den Band wurden die Stücke „Bohnen“ von Elin Rahnev, „Meersalz“ von Emil Bonev und „Das Flugzeug Flüchtling“ von Kamen Donev aufgenommen. Die Herausgeber geben nirgendwo in der Ausgabe an, welche Kriterien und Motive sie bewegt haben, genau diese drei Texte vorzustellen. Als Positiva dieser Anthologie darf man nicht nur die Qualität der Übersetzung hervorheben sondern auch den Einführungsartikel des Theaterkritikers Dimităr Černev „Über das zeitgenössische bulgarische Drama als Panorama des Wunsches“ („О савременој бугарској драми као панорами жеља”). In seinem Text stellt Černev die Entwicklung der neuesten bulgarischen Dramatik einigen Mustern aus der näheren und ferneren Vergangenheit gegenüber. „Ka- 
men Donev, Elin Rahnev und Emil Bonev sind einige der meistgespielten Dramatiker aus der jüngsten Generation." (Černev 2003: 10) Wenn man annimmt, dass dies das Kriterium ist, nach welchem die Autoren ausgewählt wurden, so lässt sich darüber diskutieren. „Meistgespielt“" ist etwas sehr Relatives und eine Sache des Standpunktes. Was die Behauptung betrifft, dass diese drei Stücke für die Vielfalt der zeitgenössischen bulgarischen Dramatik stehen, könnte ich dem Autor darin folgen. „Das unstillbare Verlangen des Kritikers zu definieren, die dramatischen Texte genremäßig einzuordnen, bestimmt Elin Rahnev als Postmodernisten, während Kamen Donev und Emil Bonev Vermittler der Massenkommunikation sind, die die Tradition des Komischen weiterentwickeln, als Schöpfer des Absurden Theaters, das sie zerstören. Sie sind klar, leserlich und nostalgisch.“ (Černev 2003: 10) Weil das bulgarische Drama in Serbien vollkommen unbekannt ist (das Gleiche gilt für das serbische Drama in Bulgarien), ist der zitierte Text ein Beweis, dass man mit den vorgestellten Stücken auf Mannigfaltigkeit hin abgezielt hat. Was die mangelnde Bekanntheit betrifft, verflechten sich hier wieder Politik und Literaturgeschichte. Bis 1944 wurden auf bulgarischen Bühnen nicht nur serbische Autoren gespielt - einer der populärsten war Branislav Nušić - sondern es gastierten wechselseitig ganze Theatertruppen mit ihren Vorstellungen sowie einzelne Schauspieler. Der bekannteste bulgarische Dramatiker in Serbien zu jener Zeit war St. L. Kostov. Später, in der Zeit der sozialistischen Existenz beider Staaten, blieben die kulturellen Beziehungen zwischen ihnen sporadisch. Die Sozialistische Republik Jugoslawien mit ihrer Neutralität und ihren unreglementierten Beziehungen außerhalb des kommunistischen Einflussbereichs wurde zum Feind erklärt; folglich war auch alles, was auf dem Gebiet der Kultur geschah, feindlich. Das Erscheinen einer Anthologie mit bulgarischen Bühnenstücken in serbischer Sprache war der Anfang eines neuen Kennenlernens der Kulturen der beiden Nachbarvölker, die in ihrer Psychologie viele gemeinsame Züge aufweisen. „Es ist sehr schön, dass diese drei Stücke den serbischen Theaterschaffenden vorgestellt werden. In ihrer Unterschiedlichkeit sind sie typische Vertreter des zeitgenössischen bulgarischen Dramas. Ich hoffe, dass sie das Interesse an dieser Dramatik hervorrufen und diese fremden Theaterräume erobern, besonders in der Nachbarschaft. Es wäre wunderbar, wenn diese Bühnenstücke Werbeagenten für den stürmischen Prozess des Verfassens von Dramen in Bulgarien werden könnten." (Černev 2003: 10) Eines der Probleme dieser Anthologie ist ihr Vertrieb. Das Buch tauchte nicht auf dem Büchermarkt in Serbien auf, erreichte aber alle Theater und rückte auf diese Weise ins Blickfeld des Interesses der Fachleute. Ermuntert durch das Interesse an Christo Bojčevs Stück „Der Oberst Vogel“, welches auf der Bühne des „Gate Theatre“ in London aufgeführt wurde, stellte der Verlag „Tantalus Books“ 2003 im englischsprachigen Raum eine neue Anthologie mit bulgarischen Bühnenstücken unter dem Titel „Der Oberst Vogel und andere Stücke“ (,THE COLONEL BIRD AND OTHER PLAYS“) vor. Herausgeberin des Sammelbandes ist Josepha Jakobson. Fast alle Übersetzungen stammen von ihr, freilich mit einer Ausnahme - „Der Oberst Vogel“ wurde von Judith Sprostranova übersetzt. Im 
Band selbst sind tatsächlich einige der interessantesten Vertreter der zeitgenössischen bulgarischen Dramatik präsent - Teodora Dimova, mit dem Stück „Neda und die Hunde“, Kamen Donev mit „Das Flugzeug Flüchtling“ sowie Elin Rahnev mit seinem Text für Theater „Off-side oder Monolog für zwei“. Der Verlag selbst bestimmt seine den Lesern vorgestellte Auswahl wie folgt: „Tantalus Books is now including this amazing play in a superb collection of a new Bulgarian drama. Written by young and upcoming playwrights these plays offer a real taste of the vibrant energy and talent in Bulgarian theatre today."(Jakobson 2003: 4). Der Verlag nutzte die Rezeption von „Der Oberst Vogel“ und besonders seine Bühneninszenierung aus, und es gelang ihm, das Interesse der Leser für die Anthologie zu wecken, indem man Kritiken aus den seriösesten englischen Zeitungen wie „The Guardian“ und „The Independent“ zitiert. Michael Billington, „,The Guardian“: „This is an extraordinary play: a politicized, Balkan One Flew Over The Cuckoo's Nest subverting all our conventional definitions of madness and sanity“. Paul Taylor, „The Independent": „For those of us who take the view that Nato's antics have amounted to a shameful farce, the exuberant staging of The Colonel Bird at the Gate comes as a kind of elating therapy." (Jakobson 2003: 4) Mit dem Erscheinen eines zweiten Sammelbandes mit bulgarischen Bühnenstücken auf dem Buchmarkt der englischsprachigen Welt zeichnet sich eine Tendenz des Interesses für die Ereignisse in der zeitgenössischen bulgarischen Dramatik ab.

In der ehemaligen Sowjetunion war die bulgarische Dramatik von den 50er bis zu den 90er Jahren des 20. Jahrhunderts sehr weit verbreitet. Nach dieser Blütezeit stagnierte das Interesse am bulgarischen Drama jedoch. Die erste Anthologie mit zeitgenössischen bulgarischen Bühnenstücken nach dieser Etappe erschien erst 2004 unter dem Titel „Zeitgenössische bulgarische Dramatik - Band 1“, („,ОВPEMEHНАЯ БОЛГАРСКАЯ ДРАМАТУРГИЯ - ВЫПУСК І“) und war eine gemeinsame Ausgabe des Bulgarischen Kultur- und Informationszentrum in Moskau und der Zeitschrift „Петербургский театралный журнал“ („St. Peterburger Theaterzeitschrift"). Herausgegeben wurde sie von der Theaterkritikerin Maja Pramatarova. Das Sammelband enthält die Stücke - „D.J.“ von Georgi Gospodinov, „Franceska“ von Konstantin Iliev, „Der Geiger des Meeres“ von Stefan Canev, „Schlangenmilch“ von Teodora Dimova, „Orchester Titanic“ von Christo Bojčev, „Der Pilz oder das Umgekehrte des Umgekehrten“ von Cvetan Marangozov, „Bohnen“ von Elin Rahnev, „Küsse von gestern“ von Jurij Dačev und „Die Wärme im November“ von Jana Dobreva. Übersetzer der Stücke sind die sehr erfahrenen und bekannten Eleonora Makarova und Elena Falkovič sowie junge Übersetzer, die sich zum ersten Mal der bulgarischen Dramatik zuwenden - Marija Širjaeva, Valentina Kornileva, Soja Šanova und Ksenia Puškina. Die Bevorzugung neuer Persönlichkeiten ist erfreulich, denn die Situation in Bezug auf die Übersetzungen aus dem Bulgarischen in Russland kann man mit der Situation in der bulgarischen Dramatik vergleichen zwischen den Klassikern und den neuen Autoren liegt zeitlich eine Kluft, die sich über Jahrzehnte erstreckt. Die Prinzipien, nach denen die Anthologie zusammenge- 
stellt wurde, sind von der Verfasserin im Vorwort wie folgt beschrieben: „Unter den angebotenen Bühnenstücken entstammen einige dem Spielplan des bulgarischen Nationaltheaters in den letzten zehn Jahren... Bei der Auswahl der Stücke für den Sammelband haben wir uns auch am Geschmack der Übersetzer bulgarischer Dramatik orientiert, welche unabhängig von den sich verändernden gesellschaftlichen Bedingungen ihr verdienstvolles Werk fortsetzen.“ (Pramatarova 2004b: 3) Tatsächlich muss man hervorheben, dass fast alle Bühnenwerke in dieser Anthologie ins Repertoire des bulgarischen Theaters aufgenommen wurden. Die Ausnahme macht das in Bulgarien unbekannte Stück „Der Geiger des Meeres“ von Stefan Canev. Dieses Werk wurde besonderer Absicht in den Band aufgenommen. Es stellt dem russischen Leser den ihm bekannten bulgarischen Dramatiker und Dichter als Autor einer postmodernen theatralischen Phantasie vor, komponiert nach Themen aus der russischen Folklore. Weitere Autoren im Sammelband sind Lyriker, die mit der Gattung des Dramas experimentieren. Einer von ihnen ist Elin Rahnev, erneut mit dem Stück „Bohnen“. Die beiden anderen Autoren können zur Zeit nur je einen Theatertext vorweisen. „Der Pilz oder das Umgekehrte des Umgekehrten“ von Cvetan Marangozov weiß mit der Uneindeutigkeit seines Sinnes und mit der Neigung des Autors zu philosophischen Paradoxen zu gefallen. Georgi Gospodinov zeigt in „D. J.“ eine postmoderne Version des Don-Juan-Mythos. Die Dramatiker Konstantin Iliev und Christo Bojčev habe ich schon bei der Analyse der beiden in Großbritannien herausgegebenen Anthologien vorgestellt. Das in diesem Band veröffentlichte Werk „Franceska" von Iliev ist eine Variante des Mythos vom hohen Wert des menschlichen „Andersseins“, das vom Autor ironisch „Okkultes Opus“ benannt wurde. „Orchester Titanic“ von Christo Bojčev ist ein Stück über die „Leute von gestern“, in dem die Verbindung des Absurden Theaters mit östlicher Philosophie einen mehrdimensionalen Raum für Träume, Clownereien und politische Assoziationen schafft. Die Bühnenstücke von Jana Dobreva, Teodora Dimova und Jurij Dačev sind einander stilistisch nahe. Sie nähern sich einander durch die dramaturgische Methode, über „belauschte Alltäglichkeit“ Sujets und Metaphern aufzubauen. Ihre Texte können verschiedenartig gelesen werden, etwa auch als Melodramen. Die Herausgeberin ist sich dessen bewusst, dass die Vielfalt der neuen bulgarischen Dramatik mit diesen Autoren alleine nicht ausgeschöpft werden kann. „Aber wir hoffen, dass die Leser die vorgestellten bulgarischen Theaterstücke in russischer Übersetzung sowohl in ihrer Selbständigkeit als auch im Kontext der allgemeinen Entwicklung des neuen bulgarischen Dramas wahrnehmen und schätzen werden. Fortsetzung folgt!“ (Pramatarova 2004b: 4) Leider gab es keine Fortsetzung. In der gleichen Reihe erschien nur noch ein Bändchen mit den interessantesten Bühnenstücken des zeitgenössischen bulgarischen Klassikers Jordan Radičkov.

Im untersuchten Zeitabschnitt erschien erstmals auch eine Anthologie in einem weiteren der Nachbarländer Bulgariens - in der Türkei. 2006 stellte der türkische Verlag „Papirüs“ die Anthologie „Bulgarische Dramen“ („BULGAR OYUNLARI“) vor. Herausgeber und Übersetzer des Sammelbandes ist Hüseyin Mevsim. Es wur- 
den vier Autoren mit fünf Stücken aufgenommen - Ivan Radoev mit „Wunder“, Stanislav Stratiev mit „Römisches Bad“, Stefan Canev mit „Jeanne d'Arc“ und Christo Bojčev mit „Der Oberst Vogel“ bzw. „Orchester Titanic“. Am Anfang der Anthologie gibt der Herausgeber eine ausführliche Übersicht über die Entwicklung der bulgarischen Dramatik in ihrer über 150jährigen Geschichte. Er bietet auch eine Übersicht über die spärlichen Inszenierungen von bulgarischen Theaterstücken auf türkischen Bühnen in der zweiten Hälfte des 20. Jahrhunderts. In dieser Hinsicht hat der Sammelband auch eine informierende Aufgabe. Aus diesem Blickwinkel betrachtet ist es logisch, dass man Autoren aufgenommen hat, die Vertreter der mittleren Generation sind. Die anthologisierten Stücke wurden zu verschiedener Zeit und unter verschiedenen Bedingungen geschrieben. „Römisches Bad“ von Stratiev entstand in den 70er Jahren des vorigen Jahrhunderts. Darin bringt der Autor seine Helden in eine absurde Situation, in der wir im Grunde genommen alle zu jener Zeit lebten. Das philosophische Drama „Jeanne d'Arc" von Stefan Canev erschien Ende der 80er Jahre und war ein Widerhall jener Entwicklung in der bulgarischen Dramatik, in der man das politische System mit Hilfe der allegorischen Sprache kritisierte. „Wunder" von Ivan Radoev erschien Anfang der 90er Jahre. Leider blieb es eines der letzten Werke dieses bedeutenden bulgarischen Dramatikers. Das Stück ist ein metaphorisches Poem über das friedliche Zusammenleben der verschiedenen ethnischen Gruppen in unserem Land. Die Theaterstücke von Christo Bojčev entstanden Mitte und Ende der 90er Jahre; ich habe sie schon im Kontext der anderen analysierten Anthologien vorgestellt. Der Band „Bulgarische Dramen“ stellt einen wesentlichen Beitrag zur Propagierung der bulgarischen Dramatik in unserem Nachbarland dar. Wenn man die komplizierten politischen Wechselbeziehungen zwischen Bulgarien und der Türkei in Betracht zieht, ist sein Erscheinen ein klares Zeichen des Interesses an unserer Kultur.

2006 erschien auf dem deutschsprachigen Büchermarkt die Anthologie „JUNGES THEATER AUS BULGARIEN“. Sie ist eine Ausgabe des österreichischen Verlages „Drava“. Herausgeber des Bandes sind der Theaterkritiker Borislav Petranov und der Übersetzer Alexander Sitzmann. Die Zusammenstellung dieses Buches wurde von der Tatsache motiviert, dass die bulgarische Dramatik in deutscher Sprache seit den Zeiten der DDR nicht mehr vorgestellt worden war. In den Sammelband aufgenommen wurden Jana Dobreva mit dem Stück „Die Wärme in November“, Teodora Dimova mit „Schlangenmilch“, Ina Božidarova mit „Saxophon“, Emil Bonev mit „Das Wort Schweigen“, Jurij Dačev mit „Salon der Tränen“ und Kalin Iliev mit „Die Grenze”. Nach welchen Kriterien die Auswahl getroffen wurde, wird von den Herausgebern folgendermaßen erklärt: „Unser Wunsch war es, Autoren vorzustellen, die sich als Dramatiker gerade in der zweiten Hälfte der 90er Jahre des 20. Jahrhunderts etabliert haben. Ihre Theaterstücke sind auf den bulgarischen Bühnen aufgeführt worden und haben sich des Interesses des Publikums erfreut. Wir sind weit entfernt von dem Gedanken, dass nur diese drei Damen und drei Herren das neue Gesicht des bulgarischen Dramas verkörpern. Aber wir sind davon überzeugt, dass 
ihre Stücke mit Sicherheit jene Vielfalt in der neuen bulgarischen Dramatik umreißen werden, von der die Rede war.“ (Petranov - Sitzmann 2006b: 7) Die Autoren in dieser Anthologie entsprechen zum Teil jenen der bisher behandelten Ausgaben, sind jedoch mit anderen Texten vorgestellt - Jurij Dačev mit dem postabsurden, auf emotioneller Basis konstruierten Stück „Salon der Tränen“ und Emil Bonev und Ina Božidarova mit ihren postmodernen Dramen. Neu in diesem Sammelband ist Kalin Iliev. Stücke von ihm wurden nicht nur auf bulgarischen Bühnen inszeniert. „Die Grenze" ist eine metaphorische Erzählung über unsere inneren und äußeren, unsere realen und erträumten Grenzen, die wir unser ganzes bewusstes Leben lang zu überwinden versuchen. Besonders wertvoll sind in dieser Anthologie die außerordentlich gelungenen Übersetzungen von Alexander Sitzmann, die es den Lesern ermöglichen, die spezifische Ausdrucksweise jedes einzelnen Autors nachzuempfinden. Doch das Projekt wird erst dann ein erfolgreiches Ende finden, wenn mindestens eines der sechs vorgestellten Stücke zur Uraufführung gelangt.

Aus dem bisher umrissenen Bild kann man einige Schlussfolgerungen ziehen:

Erstens: In einem Zeitabschnitt von vier Jahren (2002-2006) bemerkt man ein stärkeres Interesse an zeitgenössischer bulgarischer Dramatik und eine stärkere Präsenz derselben auf dem fremdsprachigen Buchmarkt. Dies ist zweifellos auch eine Folge der klaren Linie, die die Politik des Kulturinisteriums in den letzten Jahren im Hinblick auf den Vertrieb von bulgarischer Literatur außerhalb Bulgariens verfolgte.

Zweitens: Die erschienenen Anthologien lassen sich in zwei Typen teilen - in solche, die die „Klassiker“ des zeitgenössischen bulgarischen Dramas vorstellen und solche, die die neuesten Autoren des bulgarischen Theaterschaffens darstellen, d. h. jene, die sich gegen Ende der 90er Jahre des 20. Jahrhunderts durchgesetzt haben.

Drittens: Die Ziele, die sich die einzelnen Herausgeber setzen, sind verschieden und reichen von der Präsentation des Neuesten über das Wecken des während der Jahre eingeschlummerten Interesses bis zu einem gezielt informierenden Ansatz.

Als Grundtendenz lässt sich festhalten, dass die bulgarische Dramatik außerhalb Bulgariens langsam, aber sicher immer stärkere Verbreitung findet. Dieser Prozess sollte in Zukunft vertieft werden und nicht wieder abklingen. Nur so kann unsere Dramatik, die in einer „kleinen“ Sprache geschaffen ist, ihren würdigen Platz auf den Bühnen der Welt finden.

\section{L it e r a t u r}

Černev 2003: $\quad$ Д. Чернев, О савременој бугарској драми као панорами жеља, в: Ж. Хубач, Ем. Бонев (приредители), Савремена бугарска драма, Београд, 7-12

Chubač - Bonev 2003: Ж. Хубач, Ем. Бонев (приредители), Савремена бугарска драма, Београд

Jacobson 2003: J. Jacobson (ed.), The Colonel Bird and Other Plays, London

Karabinska - Jacobson 2002: A. Karabinska, J. Jacobson (ed.), Contemporary Bulgarian Plays, London

Mevsim 2006: $\quad$ H. Mevsim, Bulgar oyunlari, Istanbul

Nirvana 2005: $\quad$ Нирвана в Лондон, в: Култура 11 
Panova - Jordanov - Nikolova 2002: Sn. Panova, N. Jordanov, K. Nikolova et al. (ed.), Contemporary Bulgarian Drama 1, Sofia

Petranov - Sitzmann 2006a: B. Petranov, A. Sitzmann (Hgg.), Junges Theater aus Bulgarien, Klagenfurt

Petranov - Sitzmann 2006b: B. Petranov, A. Sitzmann, Das bulgarische Drama nach der politischen Wende, in: B. Petranov, A. Sitzmann (Hgg.), Junges Theater aus Bulgarien, Klagenfurt, 6-7

Pramatarova 2004a: М. Праматарова (сост.), Современная болгарская драматургия, Выпуск I, Москва

Pramatarova 2004b: М. Праматарова, От составителя, в: М. Праматарова, Современная болгарская драматургия, Выпуск I, Москва, 3-4

A b stract: The Bulgarian drama beyond the territory of Bulgaria in the $21^{\text {st }}$ century. Between 2002 and 2006 we witnessed an increased interest in and invasion of contemporary Bulgarian dramaturgy on the foreign book market. This can be partially attributed to the distinct guidelines regarding the distribution of Bulgarian literature outside of Bulgaria. The published anthologies can be formally divided into two types - one representing contemporary Bulgarian "classical" play-writers, and the other representing the newest names in Bulgarian drama writing, who gained recognition at the end of the 1990ies.

This development, i. e. the increase in the intensity of distribution, is the only way in which our dramaturgy, created in a "small" language, can find its honourable place on the world stage.

K e y w or d s : Bulgarian dramaturgy; Bulgarian theatre, translation, dramaturgists; playwriters, anthology

Borislav Petranov

Parkgasse 18

1030 Wien, Österreich

b_petranov@yahoo.com 
